\title{
A new approach for Supply Chain Management monitoring systems adapted to crisis
}

\author{
Quentin Schoen ${ }^{1,2}$, Sébastien Truptil ${ }^{1}$, Matthieu Lauras ${ }^{1}$, Franck Fontanili ${ }^{1}$, Aurélie \\ Conges ${ }^{1}$ \\ ${ }^{1}$ IMT Mines Albi, Campus Jarlard, \\ 81013 Albi Cedex 09, France \\ ${ }^{2}$ Etablissement Français du Sang, Avenue de Grande Bretagne, \\ 31027 Toulouse Cedex 03 \\ \{quentin.schoen, sebastien.truptil, matthieu.lauras, franck.fontanili, aurelie.conges\}@mines- \\ albi.fr
}

\begin{abstract}
Sensitive products supply chain and supply chain facing crisis management share several aspects. In both cases, several decision makers have to choose the best options most of the time under pressure, often in emergency and need to access numerous information from the field. This shared monitoring aspect put forward the visualization need to consider in each decision all the crisis potential impacts. Unfortunately, for the transportation steps we focus on, the current transport management systems do not reach these requirements. In this paper, focusing on supply chains during crisis situations, we present a new monitoring system with adapted functionalities. The added value is a connection in real time and relevant way the data from the field to the information on a shared model used to make reliable decisions. We use the French Blood Establishment supply chain to illustrate the proposition.
\end{abstract}

Keywords: Monitoring system, Supply Chain, Sensitive products, Collaboration, Real Time

\section{Introduction}

The supply chain management, as any other field, may have to face with crisis situations. The Webster's New World College Dictionary defines it as "a turning point in the course of anything; decisive or crucial time, stage, or event". We find here the idea that a crisis is decisive for the future of the given environment [1] and must have consequences. This breaking point of a stable environment may affect all the actors in it, whatever their role is. Thus, according to [2] the crisis nature may be military, politic, economic, social, technical or humanitarian.

Another aspect to consider is the time. Indeed, a crisis may occur suddenly or progressively [3], depending on the triggering event(s). These/This event(s) may have severe impacts on the considering system/environment, depending on the environment's vulnerability and the consequences of these/this event(s). For instance, a truck crash is a sudden event whereas a vehicle stuck in traffic jam is more 
progressive. Depending on the products inside (well protected or not, urgent or not), impacts may be important (valuable and/or sensitive products) or not (empty truck, not valuable products).

Being able to detect these events as soon as they happen and understand their impacts (current and potential) is one of the key elements to limit the crisis spreading and cascade effect. In order to reach this objective it is necessary to monitor the processes in real time and understand what is happening on the field. A decision support system fed with frequently updated, relevant and reliable information, would be able to help crisis units providing Common Operational Pictures (COP). The most reliable and relevant are the information provided quickly to the crisis unit, the highest are the chance to limit the impacts.

Proposal: In this situation, considering the supply chains transportation processes, our objective is to define a new way of monitoring them in crisis situation and in a larger extent a new way of monitoring sensitive products transports in general. In parallel, we describe a potential information system (IS) able to support this approach based on data collection and treatment. Our study perimeter is limited to the collection, treatment and decision support system abilities, focusing on transportation steps. The decisions broadcast to actors on the field and/or people able to concretely change parameters of the environment concerned by the crisis is out of our scope.

To reach this objective, we describe in the first section the concrete needs to respond to a crisis in supply chain. Focusing on the transportation steps, we describe in which extent Transport Management Systems could contribute to the crisis response in the second section. In the third one, a proposal is made in order to meet the described needs. Finally, we present a real use case based on our experience in the French Blood Establishment that deals with sensitive products

\section{Crisis Response from Supply Chain}

\subsection{A Response to the Crisis}

Whatever are the prevention measures to reduce the system vulnerability and the preparation plans to anticipate the crisis management, a triggering event may happen. Thus, if we did not succeed in avoiding the crisis, it is necessary to be able to respond to it.

First, [2] defines the crisis response as "special measures taken to solve problems caused by a crisis". The objective is to stop the system deviation from the "normal" or "expected" state in order to implement a new stable one. One of the main problems concerns the variety of potential actors involved in a crisis response. In order to respond to it, they may have to collaborate, each one being responsible of its area of expertise and competencies. As [4] put forward, being able to make relevant decisions, under external and internal pressure, collaborating with people we are not used to work with is not an easy task. In order to achieve this objective of collaboration and relevant decision-making, a crisis unit may be defined during crisis 
preparation steps. The role of this crisis unit is to create a decision sharing and making environment. Each person involved is responsible of its domain (vertical aspect) and of the interface with the other ones (horizontal aspect), exchanging relevant information to coordinate the response.

Thus, one of the key issues is to collect data and share between stakeholders relevant and reliable information from the crisis state. As [5] put forward, many decisions are experience and knowledge based in each domain and not shared in a formalized way. In a dynamic and unstable situation, with potential emergency pressures, this must be a challenge to meet. In the following parts, we focus on this ability to obtain relevant and reliable information in the supply chain management.

\subsection{Supply Chain Management Needs to Support Collaborative Crisis Management}

In order to make relevant decisions based on reliable information about the supply chain, the stakeholders have different needs, described below, to make decisions.

\section{Collect relevant data}

The main objective of the supply chain management is to provide the required product, in the best state, at the right place, at the right time. During a crisis, the organization scheduled has to be reconsidered and the expected events may not happen. The environment evolves quickly in unexpected ways, the system is not stable and the consequences may be important. Thus, the decision makers may become blind quickly on their own processes. The first step to find a solution is to collect data from the field in order to follow the evolutions. These data are internal, from our own processes ("Where are my vehicles? In which state are my products?"), and external from the other factors which must affect our environment (weather, traffic, emergency services, lambda individuals, etc.).

\section{Transmit and exploit reliable data}

After collecting data from the crisis state the next challenge is to transmit them to an appropriate IS and exploit them. In fact, the crisis IS has to deal with data from different sources, with different formats, putting forward different aspects of the crisis. The main challenge here is to be able to deal with these amounts of heterogeneous data, understand their meaning and build automatically relevant and reliable information. This step of data treatments allows reducing their volume and classifying them in order not to drown the stakeholders with raw data but provide required information based on them.

\section{Encourage exchanges around a shared picture}

Gathering all these information is most of the time not sufficient. Even if the information flow is understandable, these information are separated in lists, tables, and schemes, focusing on a domain. It is useful for each crisis unit stakeholders independently but does not encourage them to discuss. The information flow is reduced compared with the data flow but there is still a conceptual step to carry out in 
order to build a crisis situation overview. Creating a Common Operational Picture (COP) fosters the crisis unit members to work together from the same visualization of the crisis state; thus they understand others problematic and objectives. Moreover, this COP must be dynamic, updated frequently with new data based information, which contribute to follow and anticipate the situation.

Anticipate and foresee to support the decision-making

One of the key questions each stakeholder of a crisis unit is supposed to answer is: "knowing the current situation, what is the expected one in a near future". The need to foresee the potential next events is a key element to make decisions and evaluate their impact before the implementation.

\section{Inabilities of Transport Management Systems to Support Collaborative Crisis Management}

In order to meet the needs we described above we could try to use existing systems that deal with transportation processes. These systems manage every day thousands of containers through the world and handle anomalies, consequences of unexpected and/or unwanted transportation events. We describe below their main functionalities, failures, and some logistic trends that should contribute to prevent Transport Management Systems (TMS) to meet this challenges.

\subsection{Main Functionalities}

The Transport Management systems are applications developed from the nineties which aim to provide " "an enabling tool" for the safe and efficient operation of Freight Transport systems» [6]. Progressively the TMS evolved due to the complexification of logistics operations on one part [7] and abilities of the computing systems and the Internet on the other part. Nowadays, these applications are most of the time proposed as Software as a Service (SaaS) through the Internet and functionalities have been split in blocks activated or not. Companies, either carrier or sender, which need TMS functionalities activate the ones they want in order to fulfill their needs. They pay a rent to access them and store their data in datacenters. Cloud computing technologies and SaaS approach offer the ability to Small and Medium Size companies to access to these technologies [8]. Thus, whatever is the number of actors involved in a supply chain, the interconnectivity becomes easier to reach through normalized systems like Application Program Interface (API) and Electronic Data Interchange (EDI).

The main functionalities TMS providers offer are: (i) pick-up and Delivery areas setup, (ii) rounds setup, (iii) clients and/or subcontractors setup, (iv) packages shipment, (v) transportation and customs documents creation, (vi) rounds optimization and simulation, (vii) packages transportation steps tracking, (viii) vehicles monitoring, (ix) freight market place, quotes and order, (x) finances, 
transportation billing, (xi) reverse logistic management, (xii) performance and anomaly management, KPI, (xiii) transportation fleet management, (xiv) mobile devices setup.

These functionalities cover a broad scope of the most common needs and are split in details in different blocks activated or not. However, in case of sensitive products transportation or crisis, these systems are not relevant enough.

\subsection{Identified Problems in Case of Crisis}

For almost 30 years, the logical approach used to design TMS was to consider containers as boxes defined by a list of parameters (sender, recipient, required transportation environment, weight, permissible transportation time, etc.) and gather them in vehicles. Next, tracking the vehicles (location, state) we track the containers. Nevertheless, this approach is not fully adapted and precise enough to crisis transportation management neither sensitive products supply chain.

In fact, these products are too important to be damaged/stolen/lost, because a priori necessary to reduce the damages and contribute to recover. We should be able to know exactly where is located each product and what its state is (temperature, humidity, shocks, etc.). Consequently, the TMS real time vehicles' tracking is not precise enough and agility is limited. Indeed, during a crisis, we have to be able to make relevant decisions based on reliable facts from the field. Seeing a vehicle as a black box do not usually allow to deviate one container among the others in case of unexpected event (urgent need on a site, container damage).

Moreover, we are not able to take advantages of the multimodality opportunities that still represent a complex step because of physical and computational normalization lacks. Thus, most of the time we do not take advantages of all the transportation means opportunities [8] but use one vehicle from the sender to the recipient whereas it might not be the most effective and efficient option.

Finally, optimization rounds and allocation, which aim to increase the efficiency, cannot be fed with other data than the available means from companies the sender knows or has a contract with. Car sharing and common transport availability are not tracked. Thus, these potential loads displacement are not used in the optimization algorithms. In case of emergency, TMS are able to take advantages of just a part of all the available ability.

These for the crisis and sensitive products supply chain management issues should be exacerbated, considering the upcoming logistic environment.

\subsection{Upcoming environment}

In order to complete this analysis and anticipate future development we develop here some future logistic trends. Some of them may reinforce the TMS failures whereas some others could offer new opportunities in crisis management. 
First, the product customization is increasing as [9] foresees it. Product configurations to reach the client needs improve the capability to customize the products. The problem is the potential uniqueness of each product. In case of damage on the container or theft during the transportation steps, it would be more complicated to replace the products by identical one. In case of crisis, it would be impossible to find quickly adapted and effective solutions without gathering reliable information in real time about the locations and states of every product and container. Moreover, the additive manufacturing development encourages this customization and last miles deliveries should increase [10]. Finally, traceability requirements in particular for cold supply chains would require too this somewhat real time monitoring systems.

Meantime, several technologies are about to offer new interesting possibilities in order to deal with crisis management. The development these last years of connected devices, the Internet of Things, allows to connect any object to the Internet [8]. The development of these powerful small devices represents a remarkable opportunity to collect and transmit data from the field easily and in real time. Cloud computing development has simplified the data storage and exchanges between these devices and people. The Software as a Service [11] way of thinking permits to offer an easy, flexible and relatively cheap access to interesting functionalities to any company. Combining these technologies, we are about to create a hyper-connected world in which the problem is not how to collect data but how not to be drown by them. As we describe it in the following sections, ability to deal with big data [12] issues, powerful data treatments like machine learning, process mining... allow exploiting these data in real time to extract relevant information. As for them, autonomous vehicle development [13] and «anytime, anywhere delivery model » should create in few years a capillary distribution network, connected to monitoring systems.

This state of the art of current TMS and the expected logistic environment shows these systems would not be relevant enough to deal effectively with supply chains, which are expected to be more and more sensitive to unexpected events and should require more and more collaboration. Therefore, crisis management needs are spreading and new opportunities to meet them are about to be available. A new supply chain management IS, able to deal with them, is proposed in the next section.

\section{Proposal}

\subsection{Monitoring System}

The approach we present here focuses on the containers (and products) to be transported, considering the vehicles as "potential load displacement". In fact, considering the hyper-connected and more collaborative world we are about to live in, it seems relevant to focus on the goods to be transported and not on the means as TMS do. As data are encapsulated and send on the network with switches and connections on the Internet, we can imagine that the normalized physical network would move physically and computationally normalized containers. This idea is very 
close from the Physical Internet philosophy developed by [14]. We describe below the main functionalities to address, summarized in the figure 1 :

- Container surveillance: It is the ability to collect data about the container state, with tags in it or in its near environment, and to deduce relevant information from them. Topics like the physical and computational normalization, the container autonomy level and in which extent the decision support system is centralized or multi-agent based have still to be discussed.

- Network analysis and surveillance: While we gather data from the containers we have to gather data about all the available transportation means that are willing to load containers and their capabilities (destination, loading capacity, cost, etc.). Exploiting raw data from the field, we will be able to create reliable information about the network. On the other part, the surveillance must provide information about the network state and prevent unwanted events. Delivery center expected capacity, weather, roadwork, are some of the parameters interesting to control in real time.

- Container steering: Gathering the previous functions it would be possible to route automatically the containers on the network, step-by-step. Defining containers allocation rules to the available transportation means around them would orientate the containers to the most suitable way. In this hyperconnected environment, the ability to take advantage of the best solutions would allow efficiency and effectiveness rise. Thus, the supply chain becomes able to respond almost automatically to any unexpected event.

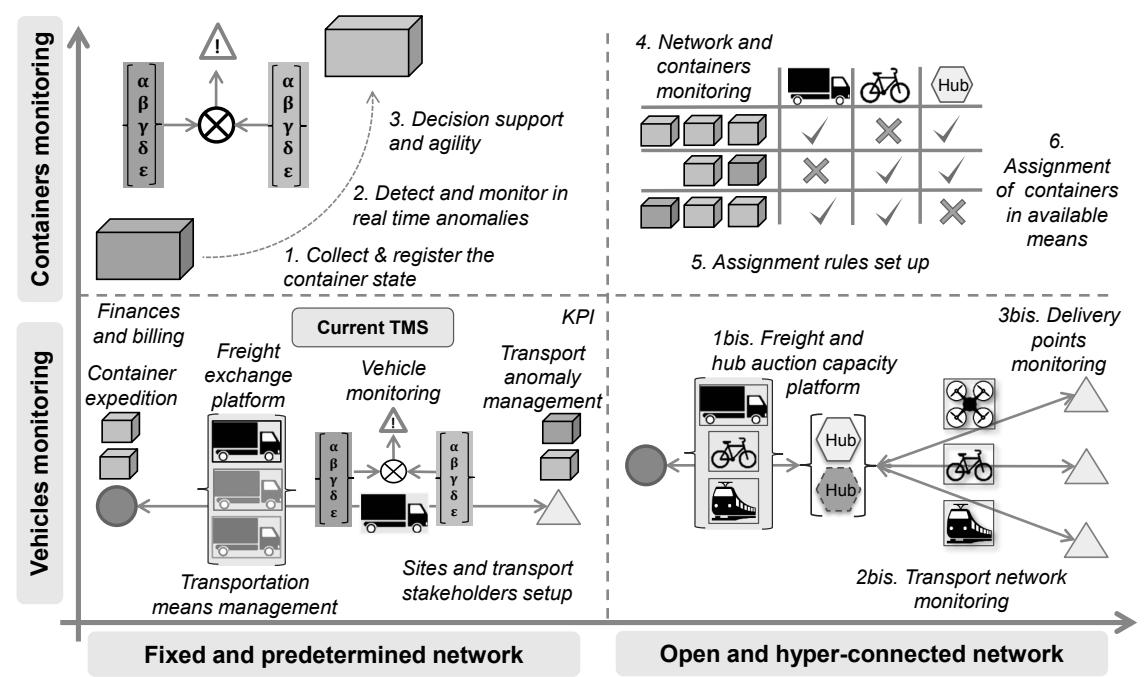

Fig. 1. Transportation monitoring systems evolution

Even if this kind of hyper-connected system may have problem in case of inability to collect enough relevant data from the field, it would meet the needs described above. 


\subsection{A New IS to Treat Big Data Issues}

In order to meet the needs we described in the first section we have to design a IS able to cope with this upcoming technological environment.

\section{Need 1: Collect relevant data.}

Whatever are the tags and technologies used to collect these data, if we want to be reactive and agile enough to reach the challenges described we need frequent updates from all of them. The Internet of Things development should allow data collection easily, from autonomous tags and sensors, mobile phones, camera, etc. Finally, we may produce too many data compared with what we really need. Thus, it brings big data constraints. In fact, as [12] define it big data may be characterized by five variables: Volume, Velocity, Variety, Veracity, and Value.

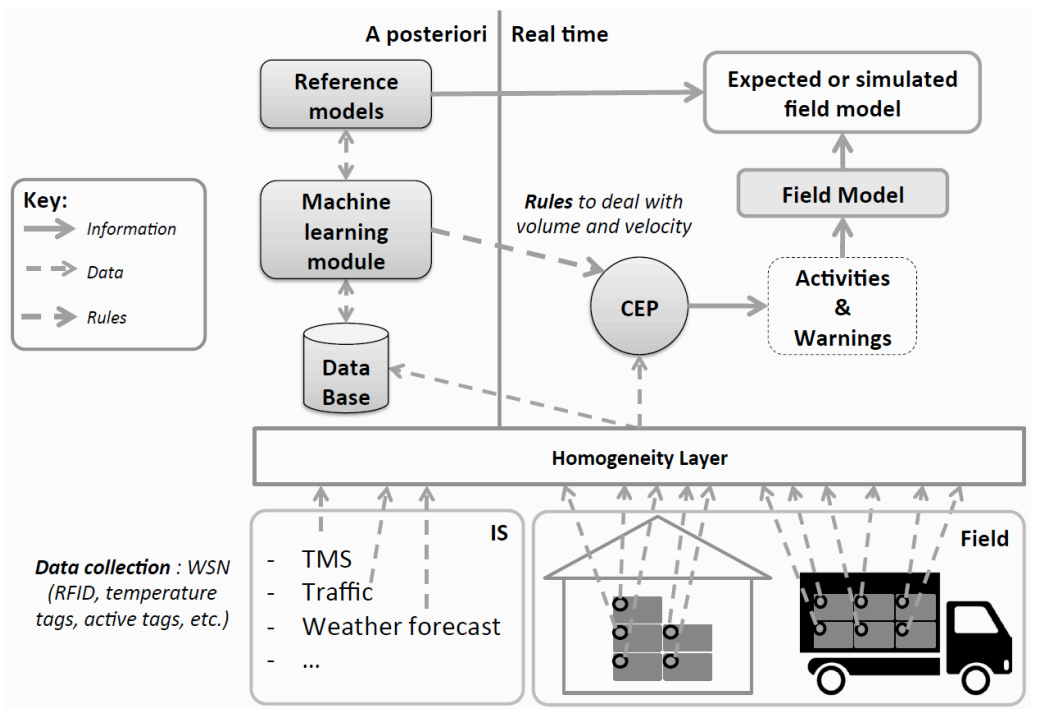

Fig. 2. Information system to support supply chain management during a crisis

\section{Need 2: Transmit and exploit reliable data}

As the figure 2 shows it, the data flow has to be filtered through a homogeneity layer that deals with data variety [15]. After that, the data flow is split in two parts. The first one consists in data storage for a posteriori treatment and the second one in real time treatments. The database is used for two purposes. First, these data represent the raw material to define the rules used to understand the data flow. For instance, combining data values from a vehicle we can deduce its state and if it represents a problem. Moreover, with a machine-learning module complex rules based on experience will be deduced in order to feed the Complex Event-Processing (CEP) module, which aims to treat in real time the data flow. Secondly, registering crisis responses these data may provide proposition in order to respond to a new one whom aspects and parameters are very close from a previous one. Machine learning analysis will enrich reference models, which contribute to help for expected model simulations. 


\section{Need 3: Encourage exchanges around a shared picture}

The second part concerns real time data treatment in which the CEP will provide reliable and relevant information based on the data from the field. Thanks to the rules, activities and warnings will be modeled and feed the field model. This model is the Common Operational Picture the crisis unit is looking for.

\section{Need 4: Anticipate and foresee to help the decision-making}

Based on the previous crises and the current field situation it is possible to foresee the next steps. Databases register how the previous crises were solved with the potential solutions, their risks and impacts. Considering the pressure of crisis decision making this ability to foresee and simulate a decision would be a major improvement.

\section{Use Case - The Blood Supply Chain}

Based on the French Blood Supply Chain, we describe below the main advantages and benefits of the defined system. The advantage of this example is that the blood supply chain is itself a sensitive one, which implies a high level of surveillance in real time. Considering the products sensitiveness and the numerous transportation steps, any unexpected event may have impacts on the products and people who need them.

\subsection{French Blood Establishment Products}

The French Blood Establishment (FBE) is the only company in France allowed dealing with the blood supply chain. The main objective of the FBE is to be able to provide anywhere, anytime, any blood product required to cure a receiver. This objective implies to collect blood from donors, check its innocuousness, separate the 3 components (plasma, red blood cells and platelets) and distribute the blood products bags the nearest to the hospitals. Due to the variety of blood products (groups and rhesus) and incompatibilities between them, it is necessary to collect the highest variety of blood types and distribute them, the closest to all the hospitals.

In France, around 10.000 pouches have to be collected every day. Consequently, every year around 40.000 one day collection sites are set up and 130 distribution sites are located the nearest to the hospitals. This variety of locations implies significant transportation steps between them.

Table 1. Lifecycles and storage temperature of the most common blood products

\begin{tabular}{lll}
\hline Products & Lifetime & Temperature \\
\hline Whole blood & Less than $48 \mathrm{~h}$ & $18-24^{\circ} \mathrm{C}(0-24 \mathrm{~h}$ post swab $)$ \\
Blood samples & Less than $48 \mathrm{~h}$ & $2-10^{\circ} \mathrm{C}(6-48 \mathrm{~h}$ post swab $)$ \\
Platelets & 5 days & $20-24^{\circ} \mathrm{C}$ shaken \\
Red blood cells & 42 days & $2-10^{\circ} \mathrm{C}$ \\
Plasma & 1 year & $<-25^{\circ} \mathrm{C}$ \\
\hline
\end{tabular}


Moreover, the preparation and checking processes require high cost equipment. They have to be highly controlled and to follow strict procedures. Thus, the FBE settled 14 preparation sites and 4 innocuousness-checking sites in France in which the pouches and samples have to pass through. In this environment, blood products transportation is required, at least from the collection site to the preparation site and checking site, then from the preparation site to the distribution site.

Finally, the blood supply chain is sensitive because the distributed products are vital for thousands of people and because of product constraints. The storage temperature and the lifecycle are the two major constraints, which affect transportation steps. We summarize in the table 1 these constraints:

This Supply Chain is a sensitive one due to the products value, their temperature and lifecycle constraints and the potential need as a matter of emergency of any product anywhere at any time. Any unexpected event may affect directly this supply chain; we develop briefly some of them in the following sub-section.

\subsection{Unexpected Events}

We can consider 4 main unexpected events that will affect the SCM. In the following situations, a crisis unit must be set up to find adapted solutions:

- A massive accident / terrorist attack raises suddenly the blood products needs in an area. This implies unexpected transports to be quickly organized.

- Traffic jam and/or bad weather conditions affect directly the transports (rounds and urgent ones). The temperature might be difficult to control if the transportation time is longer than expected.

- A major problem on a site (fire, flood, snow, inaccessibility, etc.) where high cost equipment shared between several sites and regions (samples checking) is located. This implies quick, adapted and efficient rerouting.

- In case of accident or attacks in which people are injured, the other people usually want to help and give their blood. In this situation, an unexpected rise of blood donation may affect the whole supply chain.

In order to respond to these crises, we describe in the next sub-section what would be the major improvements of the crisis management system described before.

\subsection{Illustration}

Considering this use case and the described unexpected events the figure 3 illustrates an ideal Common Operational Picture that would foster and support collaboration and help the FBE to make the best decision. Truck deviation to deliver products to the hospitals the nearest from the accident, urgent need on a site and real time truck stuck in traffic jam location are examples of this kind of abilities (cf. figure 3). 


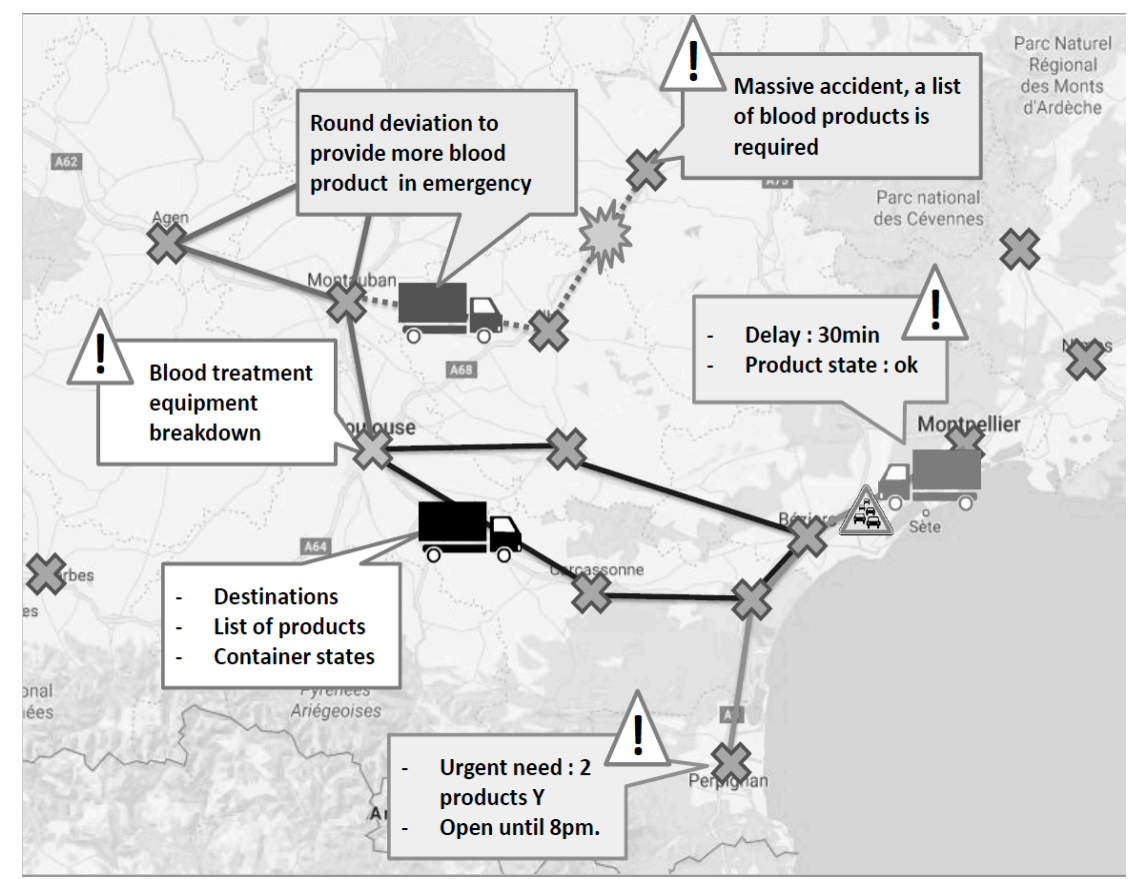

Fig. 3. Use case example of crisis management visualization

The major improvement of this kind of map is its ability to indicate on the same picture information used by all the stakeholders. This kind of visualization contributes to share an understanding of the situation and make collaborative decision.

Moreover, the truck on the left enlightened the added value of simulation to foresee the future system state. Based on machine learning analysis that feed reference models, the IS may provide an expected situation if we do not act and simulate impacts from a potential action.

\section{Conclusion}

To conclude, considering the current and foreseeable inability of Transport Management Systems to meet the needs of sensitive products supply chain and crisis management, we propose a new way of designing a transport monitoring system, data based, and putting forward the visualization. This approach would represent an important added value for supply chain management. Considering the future deployments, we have to consider several challenges. First, in this kind of collaborative platform, keeping a clear visualization for stakeholders with enough relevant information without drowning them with unnecessary ones may be difficult. Secondly, the stakeholders may be reluctant to use this kind of systems because they may feel it reduces their autonomy. Finally, the whole system is data-based, 
consequently if some data are not available for any reason (communication issues, tags breakdown, etc.) the whole system may create an inaccurate picture.

Our next tasks are now to develop concretely this kind of IS and feed it with real time transportation data. These data may come from tracking systems and devices we will use directly inside the FBE containers. The main objective is now to experiment this proposition on the field, estimate its benefits in crisis situation and discuss its failures.

\section{References}

1. Lagadec, P. La gestion des crises: outils de réflexion à l'usage des décideurs. Ediscience international, (1991)

2. Devlin, E. S. Crisis management planning and execution. CRC Press, (2006)

3. Tomasini, R. M., \& Van Wassenhove, L. N. Genetically modified food donations and the cost of neutrality: logistics response to the 2002 food crisis in Southern Africa. INSEAD case, 604-024 (2004)

4. Lagadec, P. Cellules de crise: les conditions d'une conduite efficace: gouvernements, ministères, entreprises, préfectures, administrations, municipalités, régions, médias, organisations internationales, organisations non gouvernementales, associations, syndicats. Les Éditions d'Organisation, (1995)

5. Truptil, S. Etude de l'approche de l'interopérabilité par médiation dans le cadre d'une dynamique de collaboration appliquée à la gestion de crise (Doctoral dissertation). (2011)

6. Giannopoulos, G. A. The application of information and communication technologies in transport. European Journal of Operational Research, 152(2), 302-320 (2004)

7. Marchet, G., Perego, A., \& Perotti, S. An exploratory study of ICT adoption in the Italian freight transportation industry. International Journal of Physical Distribution \& Logistics Management, 39(9), 785-812 (2009)

8. Harris, I., Wang, Y., \& Wang, H. ICT in multimodal transport and technological trends: Unleashing potential for the future. International Journal of Production Economics, 159, 88-103 (2015)

9. DHL,

http://www.dhl.com/content/dam/downloads/g0/about_us/logistics_insights/dhl_logistics_t rend_radar_2016.pdf

10. Boon, W., \& Van Wee, B. Influence of 3D printing on transport: a theory and experts judgment based conceptual model. Transport Reviews, 1-20 (2017)

11. O'sullivan, D. Software as a service: developments in supply chain IT. Logistics and Transport Focus, 9(3), 30-3 (2007)

12. Wamba, S. F., Akter, S., Edwards, A., Chopin, G., \& Gnanzou, D. How 'big data' can make big impact: Findings from a systematic review and a longitudinal case study. International Journal of Production Economics, 165, 234-246 (2015)

13. Van Meldert, B., \& De Boeck, L. Introducing autonomous vehicles in logistics: a review from a broad perspective. (2016)

14. Montreuil, B. Toward a Physical Internet: meeting the global logistics sustainability grand challenge. Logistics Research, 3(2-3), 71-87 (2011)

15. Wang, T., Truptil, S., \& Benaben, F. An automatic model-to-model mapping and transformation methodology to serve model-based systems engineering. Information Systems and e-Business Management, 15(2), 323-376 (2017) 